\title{
Using a Combination of HAADF and SE Imaging to Locate Pt Nanoparticles within a Mesoporous Silica Diesel Oxidation Catalyst
}

\author{
Hien N. Pham ${ }^{1}$, Jane Y. Howe ${ }^{2}$, Arnab Ghosh ${ }^{1}$, Matthew Melton ${ }^{1}$, Deepak Kunwar ${ }^{1}$, Eric J. Peterson ${ }^{1}$, \\ and Abhaya K. Datye ${ }^{1}$ \\ 1. Chemical \& Biological Engineering and Center for Micro-engineered Materials, University of New \\ Mexico, Albuquerque, NM 87110, USA \\ 2. Hitachi High-Technologies America Inc., Clarksburg, MD 20871, USA
}

The USDRIVE Low Temperature Oxidation Catalyst Test Protocol recommends an accelerated aging test for Pt diesel oxidation catalysts (DOCs) to be performed at $800{ }^{\circ} \mathrm{C}$ in flowing air. However, at such high temperatures, $\mathrm{Pt}$ sinters readily to form large particles due to significant vapor phase transport of $\mathrm{PtO}_{2}$. One approach we used to lower the vapor pressure of $\mathrm{PtO}_{2}$ was by alloying $\mathrm{Pt}$ with $\mathrm{Pd}$, which helped to reduce the rate of Pt sintering [1]. A second approach was to trap the mobile Pt species with an oxide such as $\mathrm{PdO}$ that reacts to form Pt-Pd bimetallic particles [2], or using $\mathrm{CeO}_{2}$, which traps Pt in atomically dispersed form [3]. Here we explore an alternative strategies, such as the use of pore confinement, to determine if it is possible to slow the vapor phase transport of $\mathrm{PtO}_{2}$. Since $\mathrm{PtO}_{2}$ vapor has negligible binding to silica [4], it provides the ideal support to study the role of pore structure. In future work, we will explore different pore structures and connectivity; for this work we focused on the approach for imaging nanoparticles in mesoporous SBA-15 silica.

The Pt diesel oxidation catalysts were synthesized via incipient wetness impregnation using mesoporous silica SBA-15 as the support, and tetra amine platinum (II) nitrate as the precursor (nominal loading 1.5 wt $\% \mathrm{Pt})$. The Pt DOCs were loaded inside a tube furnace and aged in air $(50 \mathrm{cc} / \mathrm{min})$ at $800{ }^{\circ} \mathrm{C}$ for up to $5 \mathrm{~h}$. HAADF-STEM was performed using a JEOL 2010F at $200 \mathrm{kV}$, equipped with an Oxford Aztec energy dispersive X-ray spectroscopy (EDS) system. To determine whether some of the $\mathrm{Pt}$ has remained inside the pores of SBA-15 after aging, simultaneous bright field (BF), dark field (DF) and secondary electron (SE) imaging were performed using a Hitachi probe aberration-corrected HF-5000 operated at $60 \mathrm{kV}$. This characterization is similar to our previous work in which we used a combination of HAADF-STEM and SEM to determine whether Au particles were located inside the pores of SBA-15 by changing the $\mathrm{kV}$ on the SEM [5].

Figure 1 shows DF images $(200 \mathrm{kV}$ ) of the as-prepared Pt/SBA-15 catalyst with highly dispersed Pt particles $\sim 1-2 \mathrm{~nm}$ in size. Aging in air at $800{ }^{\circ} \mathrm{C}$ for $5 \mathrm{~h}$ causes severe sintering of the catalyst, causing a bimodal distribution with the co-existence of small $(\sim 2 \mathrm{~nm})$ and large $(10 \mathrm{~nm}-100 \mathrm{~nm}) \mathrm{Pt}$ particles in $\mathrm{Pt} / \mathrm{SBA}-15$. Figure 2 shows the DF and SE images $(60 \mathrm{kV})$ of $\mathrm{Pt} / \mathrm{SBA}-15$ aged at $800{ }^{\circ} \mathrm{C}$ for (a) 1 , (b) 3 , and (c) $5 \mathrm{~h}$. The DF images show only the outlines of the large, faceted Pt particles whereas the SE images show the surface morphology, indicating that the large Pt particles are present on the outer surface of SBA-15. Moreover, the number of large Pt particles progressively increases as the catalyst is aged, and after $5 \mathrm{~h}$ a significant fraction of the $\mathrm{Pt}$ is now located on the external surface of the silica support. Although the small Pt particles shown in Figure 1 are seen after aging for $5 \mathrm{~h}$, we do not know whether they are in the pores of SBA-15. However, instead of changing the $\mathrm{kV}$ on an SEM, we did $\mathrm{BF} / \mathrm{DF} / \mathrm{SE}$ imaging using the Hitachi HF-5000 operated at $60 \mathrm{kV}$. Figure 3 shows that the BF and DF images show the presence of small Pt particles but they are not observed in the SE image, indicating that the small Pt particles are indeed retained inside the pores of SBA-15. By performing EDS from regions 
that only contain small particles, we concluded that for Pt/SBA-15, $0.5 \mathrm{wt} \% \mathrm{Pt}$ is retained in the form of small particles.

The significance of this work is that it presents a strategy to control the rate of $\mathrm{Pt}$ sintering. The cylindrical pores of SBA-15 help to retain some of the Pt within the pores, which then form small particles that co-exist with the larger particles that form outside the pores. The SE images are critical to understanding the location of the Pt and the role of pore structure in improving the sinter resistance [6].

\section{References:}

[1] C. Carrillo et al, Applied Catalysis B: Environmental 218 (2017), p. 581.

[2] C. Carrillo et al, Journal of Physical Chemistry Letters 5 (2014), p. 2089.

[3] J. Jones et al, Science 353 (2016), p. 150.

[4] P. N. Plessow and F. Abild-Pedersen, ACS Catalysis 6 (2016), p. 7098.

[5] M. T. Bore et al, Journal of Physical Chemistry B 109 (2005), p. 2873.

[6] The authors acknowledge funding from the NSF GOALI Grant CBET-1438765. Part of the STEM work was carried out at the Kuiper Core Imaging and Microscopy Facility, University of Arizona, supported by NSF and NASA.
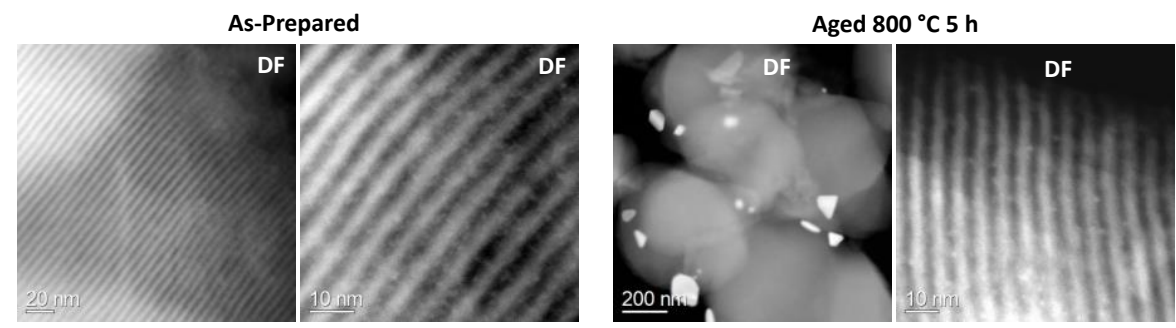

Figure 1. DF (200 kV) images of Pt/SBA-15, as prepared and after aging in air at $800{ }^{\circ} \mathrm{C}$ for $5 \mathrm{~h}$. The aged catalyst shows the co-existence of small and large Pt particles.
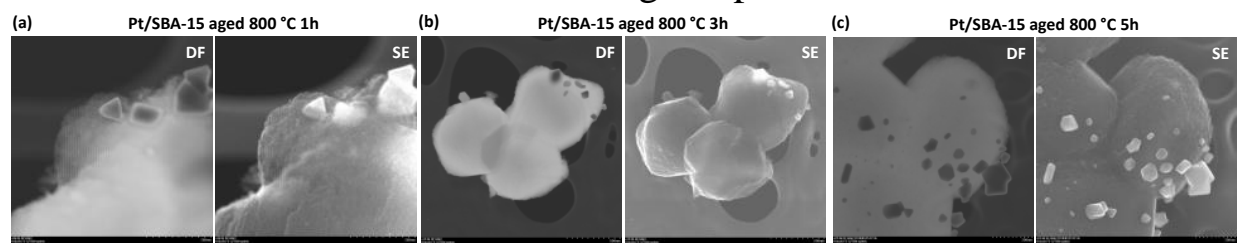

Figure 2. DF and SE images (60 kV) of Pt/SBA-15 aged in air $800{ }^{\circ} \mathrm{C}$ for (a) 1 , (b) 3 , and (c) $5 \mathrm{~h}$. The Pt initially in the pores (see Figure 1) progressively comes out of the pores, forming large Pt particles located on the surface of the silica support.

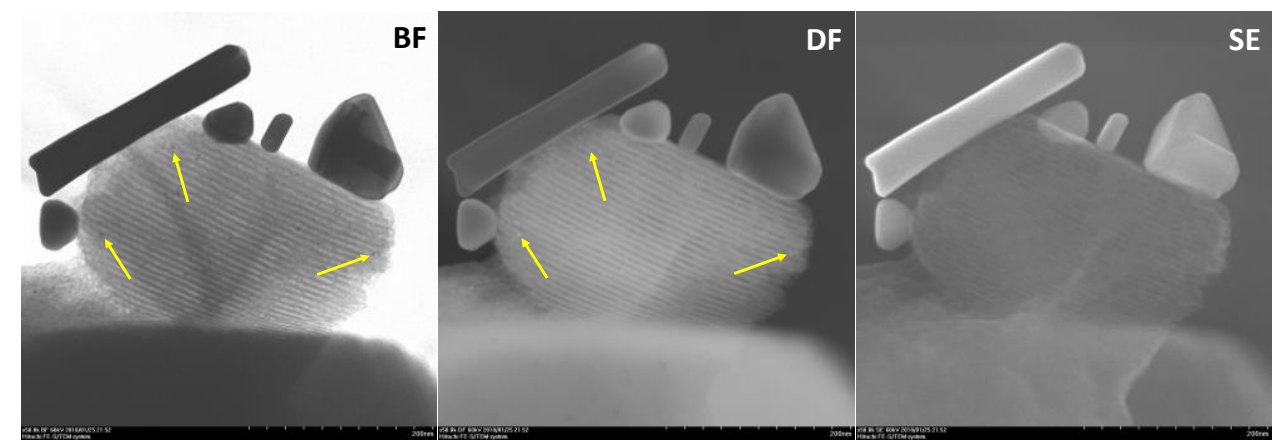

Figure 3. BF, DF and SE images $(60 \mathrm{kV})$ of Pt/SBA-15 aged in air at $800{ }^{\circ} \mathrm{C}$ for $5 \mathrm{~h}$. The small $\mathrm{Pt}$ particles are visible (arrows) in BF and DF modes, but are not seen in SE mode since they are located inside the pores. 